\title{
Electronic Raman Scattering in Twistronic Few-Layer Graphene
}

\author{
A. García-Ruiz, ${ }^{1,2}$ J. J. P. Thompson, ${ }^{1,3}$ M. Mucha-Kruczyński, ${ }^{1,4,}$ and V. I. Fal'ko ${ }^{2,5}$ \\ ${ }^{1}$ Department of Physics, University of Bath, Claverton Down BA2 7AY, United Kingdom \\ ${ }^{2}$ National Graphene Institute, University of Manchester, Booth Street East, Manchester M13 9PL, United Kingdom \\ ${ }^{3}$ Department of Physics, Chalmers University of Technology, SE-412 96 Gothenburg, Sweden \\ ${ }^{4}$ Centre for Nanoscience and Nanotechnology, University of Bath, Claverton Down BA2 7AY, United Kingdom \\ ${ }^{5}$ Department of Physics, University of Manchester, Oxford Road, Manchester M13 9PL, United Kingdom
}

(Received 21 May 2020; accepted 16 September 2020; published 5 November 2020)

\begin{abstract}
We study electronic contribution to the Raman scattering signals of two-, three- and four-layer graphene with layers at one of the interfaces twisted by a small angle with respect to each other. We find that the Raman spectra of these systems feature two peaks produced by van Hove singularities in moiré minibands of twistronic graphene, one related to direct hybridization of the Dirac states, and the other resulting from band folding caused by moiré superlattice. The positions of both peaks strongly depend on the twist angle, so that their detection can be used for noninvasive characterization of the twist, even in hBN-encapsulated structures.
\end{abstract}

DOI: 10.1103/PhysRevLett.125.197401

Twisted bilayer graphene is a van der Waals heterostructure where the relative twist of constituent atomic planes alters electronic properties of the material [1,2]. A small-angle twist in a bilayer produces a long-period moiré pattern that generates minibands for electrons with a small moiré Brillouin zone (mBZ). The minibands and gaps between them strongly depend on twist angle $\theta$ leading to Mott insulator states and superconductivity for a magic angle, $\theta \approx 1.1^{\circ}[1-11]$, where the lowest miniband appears to be almost flat. Narrow minibands also appear in $(1+2)[12,13]$ and $(1+1+1)$ [14] trilayers as well as in $(2+2)$ tetralayers [15-21]. Theoretical studies also predict the appearance of correlated and topological states in various "twistronic" graphene stacks [22-28], highlighting the need to expand the toolbox of fast and noninvasive methods for measuring twist angles in such structures.

Over the years, Raman spectroscopy emerged as a powerful tool for the characterization of graphene. Raman scattering with phonons provides information about defects, doping, strain, and the number of layers in the film [29]. Twist of graphene layers was found to lead to the resonant enhancement as well as variation of the width and position of various Raman-active phonon modes [30-37], however, with a limited accuracy in determining the twist angle. Here, we demonstrate that Raman spectroscopy of

Published by the American Physical Society under the terms of the Creative Commons Attribution 4.0 International license. Further distribution of this work must maintain attribution to the author(s) and the published article's title, journal citation, and DOI. the interband electronic excitations (ERS) [38-50] can be used to detect twist-angle-dependent features in the electronic spectrum of twistronic graphene. We study the electronic minibands and electronic contributions to the Raman spectra for few-layer graphene stacks with one of the interfaces between the layers twisted by a small angle, $\theta<2^{\circ}$, Fig. 1(a). We calculate ERS spectra of twisted bilayers $(1+1)$, trilayers $(1+2)$, and tetralayers
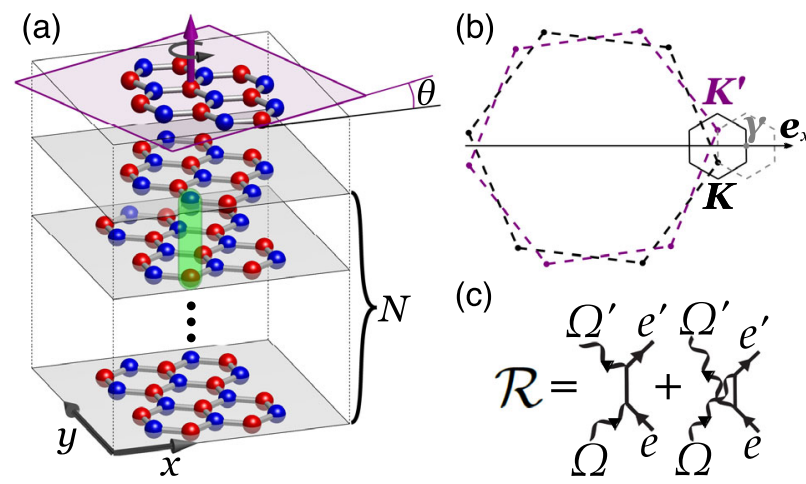

(c)

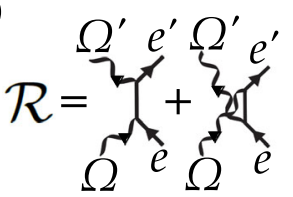

FIG. 1. (a) Sketch of $(1+N)$ twistronic graphene. The red and blue balls correspond to the two different sublattice sites in each graphene layer and green ellipse represents a dimer bonding leading to direct interlayer coupling. (b) Brillouin zones of the $N$ layer stack (dashed black line) and the top graphene (dashed purple line) with corners $\boldsymbol{K}$ (bottom layer) and $\boldsymbol{K}^{\prime}$ (top), respectively, as well as the effective moiré Brillouin zone, shown both centred around the valleys (solid black line) as the preferred choice in this Letter, and with valleys in its corners (dashed gray), centered at $\gamma$. (c) Feynman diagrams for the scattering amplitudes $\mathcal{R}$ contributing to the electronic Raman features discussed in this Letter. 
$[(1+3)$ and $(2+2)]$ and show that these are formed by transitions from the $n$th valence to the $n$th conduction moiré superlattice (mSL) miniband and feature two spectral peaks. One, at a lower Raman shift, is caused by the resonant hybridization of electronic states of the two few-layer graphene crystals separated by the twisted interface forming the lowest-energy minibands [51,52]. Another higher-energy peak is due to the anticrossing of bands, backfolded by mSL. Both peaks are related to van Hove singularities in the mSL minibands and involve electronic excitations different from those responsible for the optical absorption [53,54]. We trace the peaks positions as a function of the twist angle and estimate their quantum efficiency, $I \sim 10^{-11}$, to be in the measurable range [38-45].

To model twistronic graphene, we use a hybrid $k \cdot p$ theory-tight-binding model, where we describe electrons' states in each flake using the $k \cdot p$ expansion around $\pm \boldsymbol{K}$ and $\pm \boldsymbol{K}^{\prime}$ Brillouin zone corners of the bottom and anticlockwise rotated (by angle $\theta$ ) top crystal, respectively [see Fig. 1(b)], and the interlayer hybridization using tunneling Hamiltonian [52,55],

$$
\begin{aligned}
\hat{H}=\left[\begin{array}{ccc|cccc}
\ddots & \vdots & \vdots & \vdots & \vdots & \vdots & . \\
\ldots & \hat{H}_{+} & \hat{T}_{t} & 0 & 0 & 0 & \ldots \\
\ldots & \hat{T}_{t}^{\dagger} & \hat{H}_{+} & \hat{\mathcal{T}} & 0 & 0 & \ldots \\
\hline \ldots & 0 & \hat{\mathcal{T}}^{\dagger} & \hat{H}_{-} & \hat{T}_{b} & 0 & \ldots \\
\ldots & 0 & 0 & \hat{T}_{b}^{\dagger} & \hat{H}_{-} & \hat{T}_{b^{\prime}} & \ldots \\
\ldots & 0 & 0 & 0 & \hat{T}_{b^{\prime}}^{\dagger} & \hat{H}_{-} & \ldots \\
\ddots & \vdots & \vdots & \vdots & \vdots & \vdots & \ddots
\end{array}\right], \\
\hat{H}_{ \pm}=v \boldsymbol{\sigma}_{\xi} \cdot\left(\boldsymbol{p} \mp \xi \hbar \frac{\theta}{2} \boldsymbol{K} \boldsymbol{e}_{y}\right) .
\end{aligned}
$$

Here, $\sigma_{\xi}=\left(\xi \sigma_{x}, \sigma_{y}\right)$ and $\sigma_{x}, \sigma_{y}, \sigma_{z}$ are sublattice Pauli matrices for electrons within each monolayer, and the vertical and horizontal lines mark the twisted interface. Momentum $\boldsymbol{p}=\left(p_{x}, p_{y}\right)$ is measured from the center of the valley $\xi \boldsymbol{K}$ in the bottom flake (with valley index $\xi= \pm 1), K=|\boldsymbol{K}|, \boldsymbol{e}_{y}$ is a unit vector along wave vector $y$ axis, and $v$ is the monolayer Dirac velocity. To describe the $(M Y+N X)$ twistronic system, where $M$ and $N$ are the thicknesses of the stack above and below the twisted interface and $X$ and $Y$ describe the stacking of the corresponding crystal $[X=B$ for Bernal and $X=R$ for rhombohedral trilayer, $X=A B$ and $Y=A B$ or $Y=$ $B A$ for $(2+2)$ structures], one needs to truncate $\hat{H}$ at $M$ blocks in the top left and $N$ in the bottom right parts. Coupling across the twisted interface is described by $[52,55]$

$$
\begin{aligned}
\hat{\mathcal{T}} & =t\left(\hat{\mathcal{T}}_{0}+\hat{\mathcal{T}}_{1} e^{i \xi \boldsymbol{G}_{1} \cdot \boldsymbol{r}}+\hat{\mathcal{T}}_{2} e^{i \xi \boldsymbol{G}_{2} \cdot \boldsymbol{r}}\right), \\
\hat{\mathcal{T}}_{n} & =1+\cos \left(\frac{2 \pi n}{3}\right) \sigma_{x}+\xi \sin \left(\frac{2 \pi n}{3}\right) \sigma_{y},
\end{aligned}
$$

where $\boldsymbol{G}_{n} \approx\left[(-1)^{n} \frac{1}{2},(\sqrt{3} / 2)\right] \sqrt{3} \theta K$ and $t \approx 110 \mathrm{meV}$ [55]. Coupling $\hat{\mathcal{T}}$ is responsible for the mSL effects and defines the hexagonal mBZ, chosen as in Fig. 1(b). Interlayer couplings across the nontwisted interfaces are set using

$$
\begin{aligned}
& \hat{T}\left( \pm \frac{\theta}{2}\right)=\left[\begin{array}{cc}
-v_{4} \hat{\pi}^{\dagger}\left( \pm \frac{\theta}{2}\right) & v_{3} \hat{\pi}\left( \pm \frac{\theta}{2}\right) \\
\gamma_{1} & -v_{4} \hat{\pi}^{\dagger}\left( \pm \frac{\theta}{2}\right)
\end{array}\right], \\
& \hat{\pi}\left( \pm \frac{\theta}{2}\right)=\left[\xi p_{x}+i p_{y} \mp i \xi \hbar \frac{\theta}{2} K\right],
\end{aligned}
$$

where $\gamma_{1}=0.39 \mathrm{eV}, v_{3} \approx 0.11 v$, and $v_{4} \approx 0.015 v$ [56]. For a $(1+2)$ trilayer, $\hat{T}_{b}=\hat{T}[-(\theta / 2)]$. For a $(1+3 R)$ stack, $\hat{T}_{b}=\hat{T}_{b^{\prime}}=\hat{T}(-\theta / 2)$. For a $(1+3 B)$ tetralayer, $\hat{T}_{b}=\hat{T}(-\theta / 2)$ and $\hat{T}_{b^{\prime}}=\hat{T}^{\dagger}(-\theta / 2)$. For a $(2 A B+2 A B)$ structure, $\quad \hat{T}_{t}=\hat{T}(\theta / 2), \quad \hat{T}_{b}=\hat{T}(-\theta / 2)$, while for $(2 A B+2 B A), \hat{T}_{b}=\hat{T}(-\theta / 2), \hat{T}_{t}^{\dagger}=\hat{T}(\theta / 2)$.

In the electronic Raman scattering, photon of energy $\Omega$ carrying vector potential $\boldsymbol{A}=\sqrt{\left(\hbar / 2 \epsilon_{0} \Omega\right)}\left[\boldsymbol{l} e^{i(\boldsymbol{q} \cdot \boldsymbol{r}-\Omega t) / \hbar} \hat{b}_{\boldsymbol{q}, \boldsymbol{l}}+\right.$ H.c.] $\left(\hat{b}_{\boldsymbol{q}, l}\right.$ annihilates a photon with momentum $\boldsymbol{q}$ and polarization $\boldsymbol{l}$ ), arrives at the sample (here, we assume normal incidence of light). This photon scatters to another one with energy $\tilde{\Omega}=\Omega-\omega$, momentum $\tilde{\boldsymbol{q}}$, and polarization $\tilde{l}$, leaving behind an electron-hole pair with energy $\omega$. In contrast to classical plasmas, where the amplitude of such process is controlled by contact interaction, in graphene, the dominating contribution comes from a twostep process, described by the Feynman diagrams shown in Fig. 1(c). It corresponds to absorption (emission) of a photon with energy $\Omega(\tilde{\Omega})$ transferring an electron with momentum $\boldsymbol{p}$ from an occupied state in the valence band into a virtual intermediate state (energy is not conserved at this stage), followed by emission (absorption) of a second photon with energy $\tilde{\Omega}(\Omega)[38,39]$. The amplitude of this process is [45]

$$
\mathcal{R}=i \frac{(e \hbar v)^{2}}{\epsilon_{0} \Omega^{2}}\left(\boldsymbol{l} \times \tilde{\boldsymbol{l}}^{*}\right)_{z} \hat{I}_{M+N} \otimes \sigma_{z}
$$

where $\hat{I}_{M+N}$ is a $(M+N) \times(M+N)$ unit matrix. The main contribution to the Raman signal comes from $n^{-} \rightarrow n^{+}$miniband transitions, where $n^{s}$ denotes the $n$th miniband on the valence $(s=-1)$ or conduction $(s=1)$ side. The structure of the two-photon coupling vertex in Eq. (3) [38] suggests that, for circularly polarized photons, both the incoming and outgoing light have the same polarization. In turn, for linearly 
polarized light, the incoming and outgoing light carries perpendicular polarization (so-called linear cross polarization). To compare, the phonon $G$-line is observed in both parallel and perpendicular linear polarization geometries whereas for circular polarization the incoming and outgoing photons have opposite polarization $[42,43,47-$ 49,57]. The overall line shape $g(\omega)$ of inelastic photon scattering with Raman shift $\omega$ is given by

$$
\begin{aligned}
g(\omega)= & \frac{1}{c} \int \frac{d \tilde{\boldsymbol{q}} w(\omega)}{(2 \pi \hbar)^{3}} \delta(\tilde{\boldsymbol{\Omega}}-c|\tilde{\boldsymbol{q}}|) \approx \frac{\Omega^{2}}{(2 \pi \hbar)^{3} c^{4}} w(\omega), \\
w(\omega)= & \frac{2}{\pi \hbar^{3}} \sum_{n^{s}, m^{s^{\prime}}} \int d \boldsymbol{p}\left|\left\langle\boldsymbol{p}, m^{s^{\prime}}|\mathcal{R}| \boldsymbol{p}, n^{s}\right\rangle\right|^{2} \\
& \times f_{\boldsymbol{p}, n^{s}}\left(1-f_{\boldsymbol{p}, m^{s^{s}}}\right) \delta\left(\epsilon_{\boldsymbol{p}, m^{s^{\prime}}}-\epsilon_{\boldsymbol{p}, n^{s}}-\omega\right),
\end{aligned}
$$

where $f_{p, n^{s}}$ is the occupation factor of a state $\left|\boldsymbol{p}, n^{s}\right\rangle$ with momentum $\boldsymbol{p}$ and energy $\epsilon_{\boldsymbol{p}, n^{s}}$ in the band $n^{s}$.

In Fig. 2 we present the ERS intensity map for a $(1+1)$ twisted bilayer graphene with $0.8^{\circ}<\theta<2^{\circ}$, neglecting its atomic reconstruction [58]. Two bright features stand out in this plot, corresponding to transitions between the minibands $1^{-} \rightarrow 1^{+}$and $2^{-} \rightarrow 2^{+}$. For two selected cuts at $\theta=1.8^{\circ}$ and $\theta=1.1^{\circ}$, the peaks come from transitions indicated by arrows in the corresponding miniband plots. The first peak, $1^{-} \rightarrow 1^{+}$, is due to transitions from (or to) flat regions of electronic dispersion resulting from hybridization of the Dirac cones of the two layers (the Dirac points can be identified in the dispersion for $\theta=1.8^{\circ}$ as touching points of the minibands shown in red), which are responsible for a van Hove singularity (vHs) in the density of states [51,52] for larger twist angles. Note that due to the chiral nature of graphene electrons, the vHs is not positioned on the line connecting the cones but is shifted off it in the opposite directions in the conduction and valence bands (as indicated by black arrows in Fig. 2 for $\theta=1.8^{\circ}$ ). The inset below the $\theta=1.8^{\circ}$ miniband structure highlights in yellow parts of the $\mathrm{mBZ}$ that contribute to the $1^{-} \rightarrow 1^{+}$ peak. As the twist angle is decreased, this ERS peak moves to lower energies. The second peak, $2^{-} \rightarrow 2^{+}$, is due to the transitions between the flat regions of the second valence and conduction minibands, indicated by orange arrows in the $\theta=1.1^{\circ}$ miniband plot. Its intensity comes from the $\mathrm{mBZ}$ section painted in yellow in the leftmost inset below the ERS map. The other two insets show the real-space distribution of the saddle point states across the moiré supercell in the top and bottom monolayers.

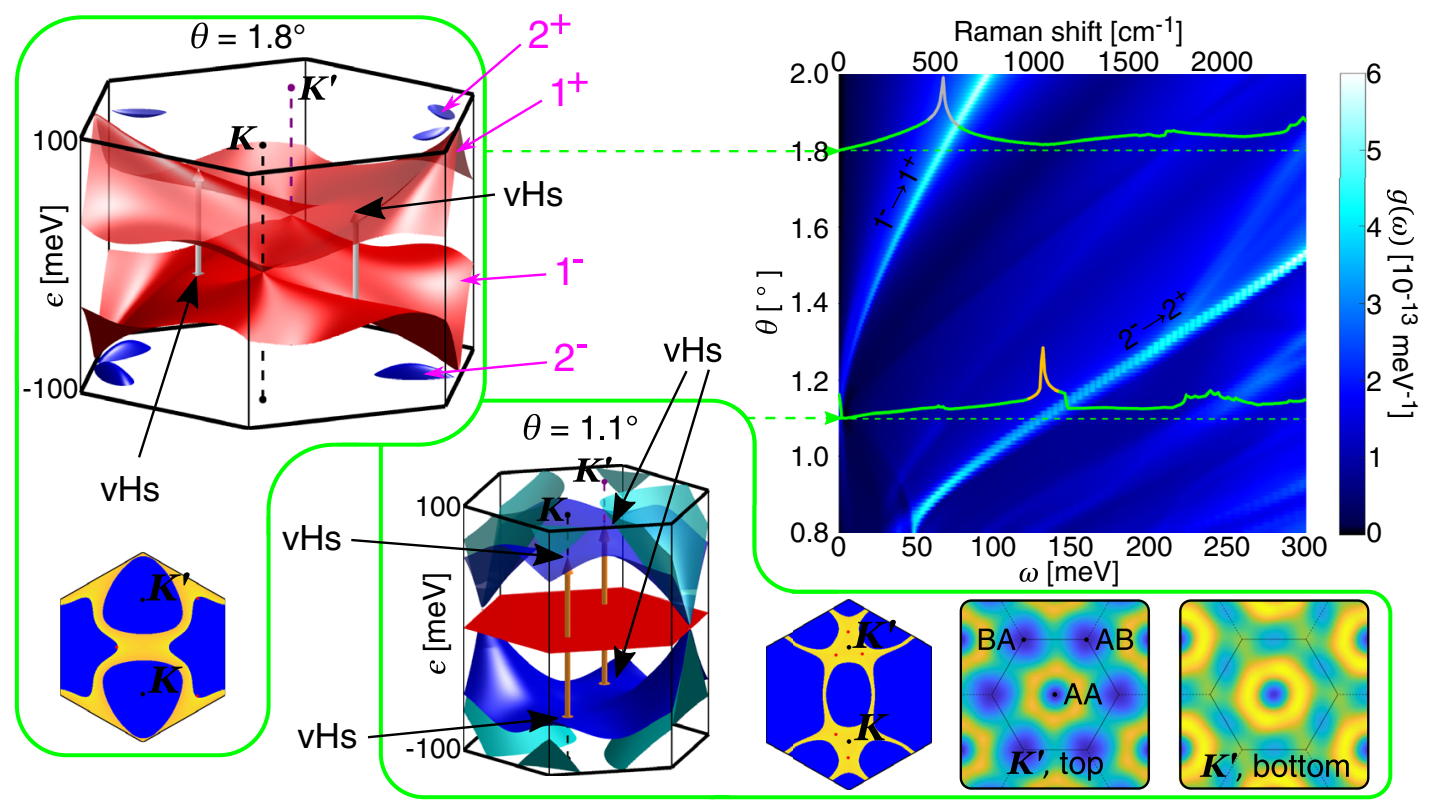

FIG. 2. Left: Electronic miniband structures of twisted bilayer graphene for $\theta=1.8^{\circ}$ and $\theta=1.1^{\circ}$ across the corresponding mBZ (solid black hexagons). The dashed lines indicate the positions of the valleys $\boldsymbol{K}$ (bottom layer) and $\boldsymbol{K}^{\prime}$ (top) within the $\mathrm{mBZ}$. The pink arrows identify the minibands and the black arrows point to saddle points which give rise to van Hove singularities (vHs). Right: ERS intensity map for $0.8^{\circ}<\theta<2^{\circ}$. The green solid lines show cuts for the angles $\theta=1.8^{\circ}$ and $\theta=1.1^{\circ}$, indicated with the green dashed lines. The gray and orange peaks mark spectral features corresponding to excitations indicated with vertical arrows of the same color in the miniband dispersions which involve dispersion saddle points. The hexagonal insets next to band structures show in yellow mBZ regions which contribute to the $1^{-} \rightarrow 1^{+}$ERS peak for $\theta=1.8^{\circ}$ and $2^{-} \rightarrow 2^{+}$peak for $\theta=1.1^{\circ}$; red dots indicate positions of dispersion saddle points on the valence side. The additional two insets for $\theta=1.1^{\circ}$ depict real space distribution of the saddle points wave function in the top or bottom layers; black solid lines mark boundaries of the moiré supercell and the letters indicate local stacking. 

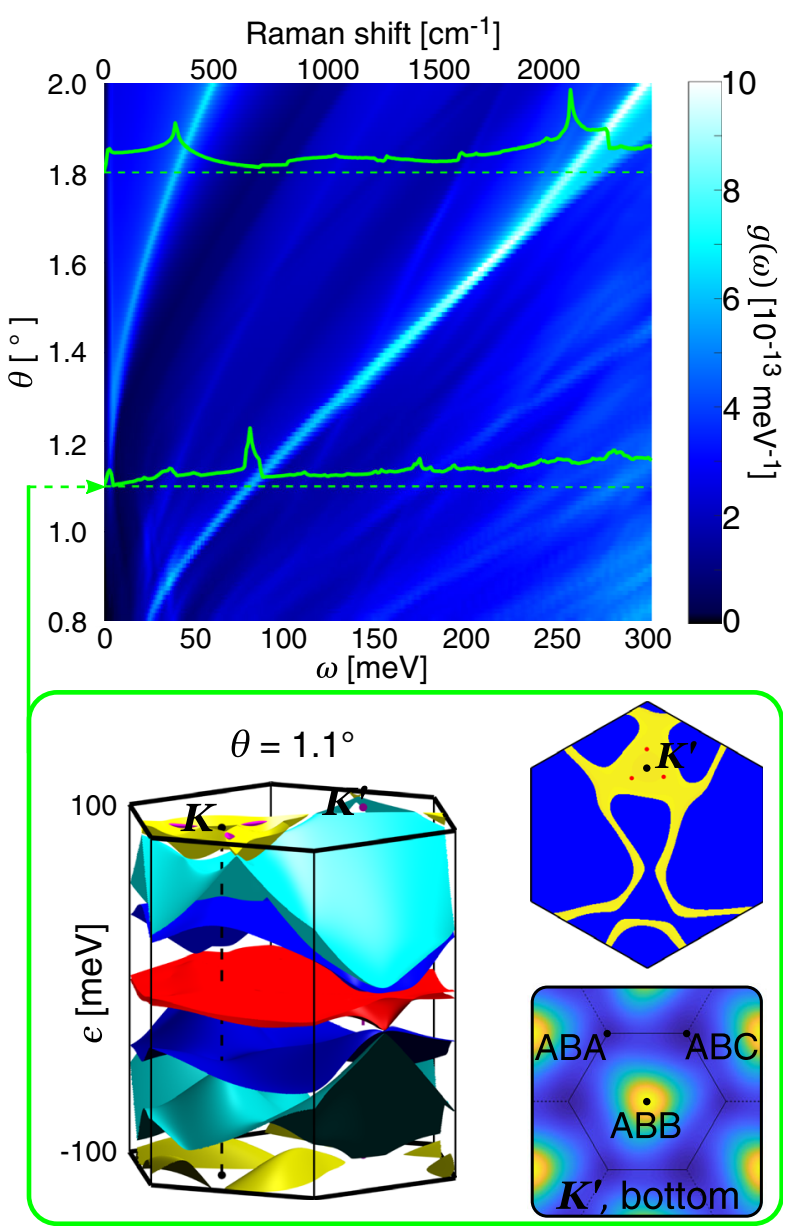

FIG. 3. Top: ERS intensity map for $(1+2)$ graphene. The green dashed lines indicate twist angles $\theta=1.8^{\circ}$ and $\theta=1.1^{\circ}$ and the green solid lines show the ERS spectra for these angles. Bottom: Miniband structure for the $(1+2)$ stack for $\theta=1.1^{\circ}$. The top inset shows in yellow the $\mathrm{mBZ}$ regions which contribute to the $2^{-} \rightarrow 2^{+}$ERS peak; red dots indicate positions of dispersion saddle points on the valence side. The saddle points wave function is predominantly located in the bottom layer and its real space distribution is presented in the bottom inset; black solid lines mark boundaries of the moiré supercell and the letters indicate local stacking (from the bottom layer to the top).

In Fig. 3, we show the ERS intensity map for $(1+2)$ twistronic graphene and an exemplary miniband structure for $\theta=1.1^{\circ}$. Similarly to $(1+1)$ graphene, the dominant contributions come from the $1^{-} \rightarrow 1^{+}$and $2^{-} \rightarrow 2^{+}$electronic transitions. The two peaks also have the same origins: the first one is due to direct hybridization of the monolayer and bilayer states while the second is formed by states backfolded by moire superlattice. However, for a given twist angle, the peaks appear at lower Raman shifts than in the $(1+1)$ structure. This is because the unperturbed dispersion in bilayer is flatter than in a monolayer-as a consequence, the anticrossings vHs form at lower energies than in a $(1+1)$ stack.

In Figs. 4(a) and 4(b), we plot ERS maps of two monolayer-on-trilayer structures, $(1+3 B)$ and $(1+3 R)$. Bernal-stacked trilayer graphene hosts both a bilayerand monolayerlike low-energy bands [59]. Both of these hybridize with the states of the top monolayer to form the first miniband and contribute to the $1^{-} \rightarrow 1^{+}$ERS peak. The next peak, marked as $\left(1+2^{\prime}\right)$ in Fig. 4(a), is due to the anticrossing of backfolded bilayerlike and top monolayer bands. Another peak, $\left(1+1^{\prime}\right)$, is due to an anticrossing of the backfolded top monolayer band and monolayerlike band of the trilayer. In contrast, rhombohedral trilayers only host one low-energy band, with a flat dispersion in the vicinity of the valley center [60]. This low-energy band is localized on the top and bottom surfaces of the crystal (representing surface states generic for rhombohedral graphitic films [61-63]) and is significantly affected by $\mathrm{mSL}$, leading to a pair of clear spectral features, Fig. 4(b), as in $(1+1)$ and $(1+2)$ twistronic graphenes (in the Supplemental Material, we describe a simple model that can be used to determine approximate positions of these ERS peaks [64]).

In Figs. 4(c) and 4(d), we present ERS spectra for two structurally inequivalent double-bilayer stacks, $(2 A B+2 B A)$ and $(2 A B+2 A B)$. The details of their lattice structure and exemplary miniband dispersions are shown in the Supplemental Material [64]. The miniband spectra of $A B / A B$ and $A B / B A$ tetralayers are nearly identical, leading to the $1^{-} \rightarrow 1^{+}$and $2^{-} \rightarrow 2^{+}$ERS peaks at the same energies. However, due to the difference in the wave functions, the intensity of the $1^{-} \rightarrow 1^{+}$is much higher for $(2 A B+2 A B)$ than for $(2 A B+2 B A)$ tetralayers (see Supplemental Material [64]).

Overall, electronic Raman scattering spectra of twistronic graphene contain characteristic features related to the van Hove singularities of moiré superlattice minibands, which depend on the twist angle between the layers. Currently, accurate determination of the twist angle, especially in the small-angle regime, requires time-consuming microscopic investigations of the moiré pattern, or magnetotransport measurements at cryogenic temperatures. We suggest that, based on our results, calibration of the positions of the Raman features in the structures with known $\theta$ would allow to identify the orientations of the component crystals in other samples, enabling a noninvasive method for measuring the twist angle, even in structures encapsulated with other materials, where the graphene or graphene moiré pattern is 
(a)

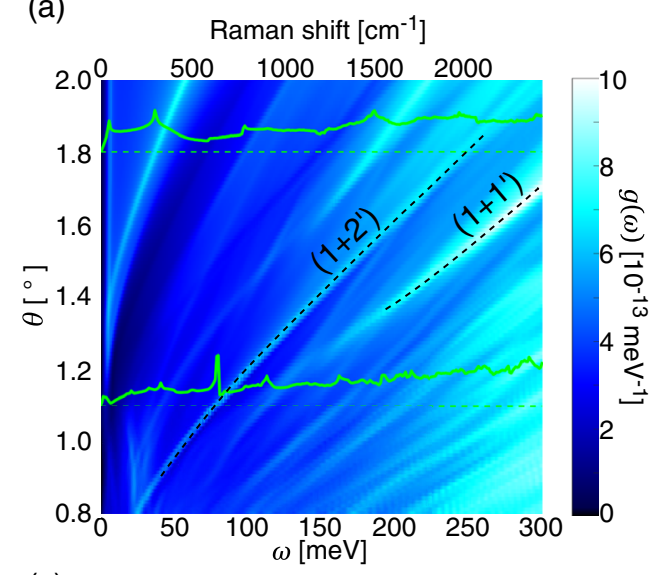

(c)

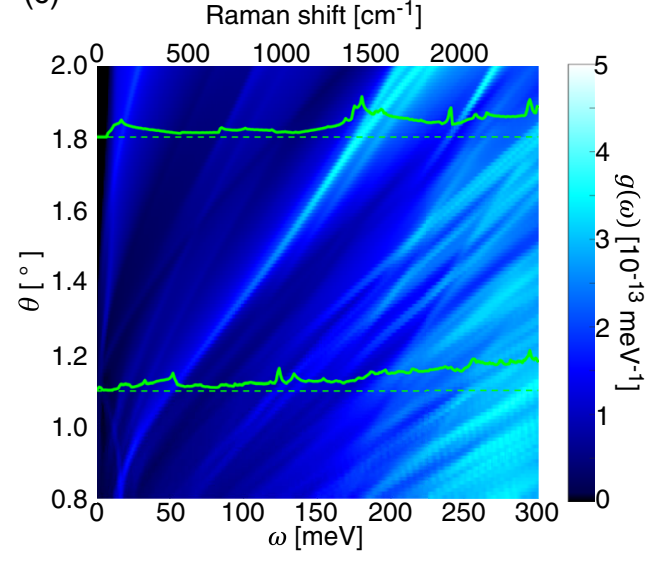

(b)

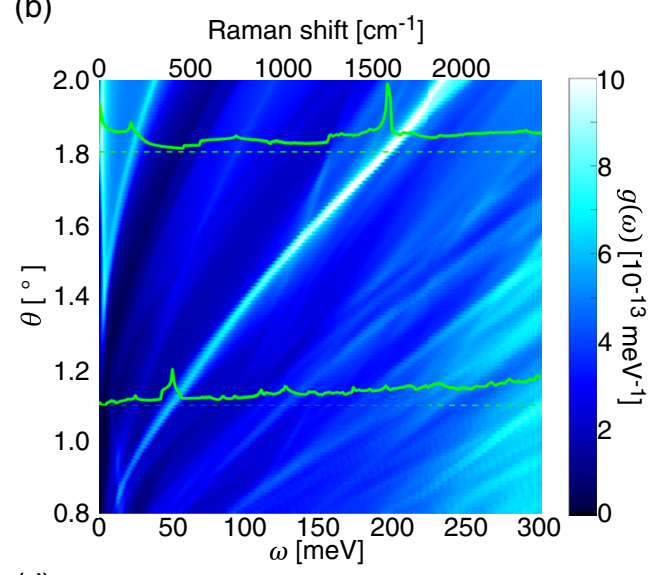

(d)

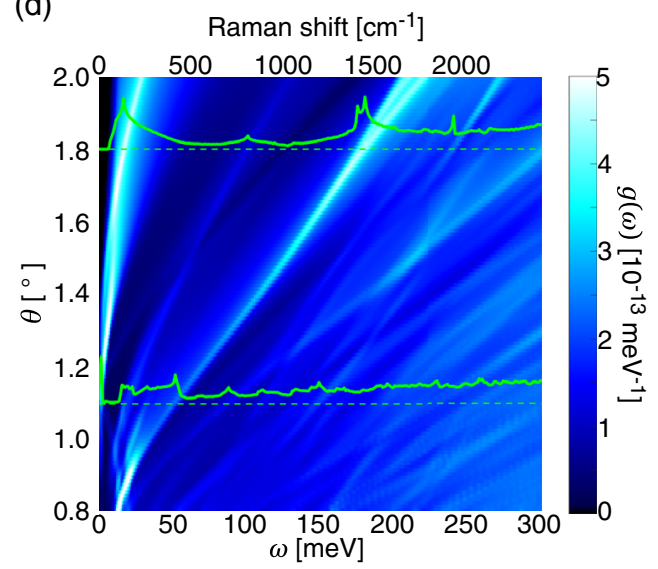

FIG. 4. Comparison of ERS intensity maps for (a) $(1+3 B)$, (b) $(1+3 R)$, (c) $(2 A B+2 B A)$, and (d) $(2 A B+2 A B)$ tetralayers. Green solid lines show the Raman spectra for twist angles $\theta=1.8^{\circ}$ and $\theta=1.1^{\circ}$.

not directly accessible for tunnelling spectroscopy studies $[1,2,5,6,9-21]$.

This work has been supported by the UK Engineering and Physical Sciences Research Council (EPSRC) through the Centre for Doctoral Training in Condensed Matter Physics (CDT-CMP), Grant No. EP/L015544/1, EPSRC Grants No. EP/M507982/1, No. EP/S019367/1, No. EP/ P026850/1, and No. EP/N010345/1, the European Graphene Flagship Core3 project (EC Grant Agreement No. 881603), European Research Council Synergy Grant Hetero2D and Lloyd's Register Foundation Nanotechnology Programme. M. M.-K. also acknowledges funding through the University of Bath International Research Funding Scheme.

*m.mucha-kruczynski@bath.ac.uk

[1] Y. Cao, V. Fatemi, S. Fang, K. Watanabe, T. Taniguchi, E. Kaxiras, and P. Jarillo-Herrero, Unconventional superconductivity in magic-angle graphene superlattices, Nature (London) 556, 43 (2018).

[2] Y. Cao, V. Fatemi, A. Demir, S. Fang, S. L. Tomarken, J. Y. Luo, J. D. Sanchez-Yamagishi, K. Watanabe, T. Taniguchi,
E. Kaxiras, R. C. Ashoori, and P. Jarillo-Herrero, Correlated insulator behaviour at half-filling in magic-angle graphene superlattices, Nature (London) 556, 80 (2018).

[3] Y. Xie, B. Lian, B. Jack, X. Liu, C.-L. Chiu, K. Watanabe, T. Taniguchi, B. A. Bernevig, and A. Yazdani, Spectroscopic signatures of many-body correlations in magic-angle twisted bilayer graphene, Nature (London) 572, 101 (2019).

[4] Y. Choi, J. Kemmer, Y. Peng, A. Thomson, H. Arora, R. Polski, Y. Zhang, H. Ren, J. Alicea, G. Refael, F. von Oppen, K. Watanabe, T. Taniguchi, and S. Nadj-Perge, Electronic correlations in twisted bilayer graphene near the magic angle, Nat. Phys. 15, 1174 (2019).

[5] E. Codecido, Q. Wang, R. Koester, S. Che, H. Tian, R. Lv, S. Tran, K. Watanabe, T. Taniguchi, F. Zhang, M. Bockrath, and C. N. Lau, Correlated insulating and superconducting states in twisted bilayer graphene below the magic angle, Sci. Adv. 5, eaaw9770 (2019).

[6] S. L. Tomarken, Y. Cao, A. Demir, K. Watanabe, T. Taniguchi, P. Jarillo-Herrero, and R. C. Ashoori, Electronic Compressibility of Magic-Angle Graphene Superlattices, Phys. Rev. Lett. 123, 046601 (2019).

[7] Y. Jiang, X. Lai, K. Watanabe, T. Taniguchi, K. Haule, J. Mao, and E. Y. Andrei, Charge order and broken rotational symmetry in magic-angle twisted bilayer graphene, Nature (London) 573, 91 (2019). 
[8] A. Kerelsky, L. J. McGilly, D. M. Kennes, L. Xian, M. Yankowitz, S. Chen, K. Watanabe, T. Taniguchi, J. Hone, C. Dean, A. Rubio, and A. N. Pasupathy, Maximized electron interactions at the magic angle in twisted bilayer graphene, Nature (London) 572, 95 (2019).

[9] X. Lu, P. Stepanov, W. Yang, M. Xie, M. A. Aamir, I. Das, C. Urgell, K. Watanabe, T. Taniguchi, G. Zhang, A. Bachtold, A. H. MacDonald, and D. K. Efetov, Superconductors, orbital magnets and correlated states in magic-angle bilayer graphene, Nature (London) 574, 653 (2019).

[10] M. Yankowitz, S. Chen, H. Polshyn, Y. Zhang, K. Watanabe, T. Taniguchi, D. Graf, A. F. Young, and C. R. Dean, Tuning superconductivity in twisted bilayer graphene, Science 363, 1059 (2019).

[11] P. Stepanov, I. Das, X. Lu, A. Fahimniya, K. Watanabe, T. Taniguchi, F. H. L. Koppens, J. Lischner, L. Levitov, and D. K. Efetov, Untying the insulating and superconducting orders in magic-angle graphene, Nature (London) 583, 375 (2020).

[12] S. Chen, M. He, Y.-H. Zhang, V. Hsieh, Z. Fei, K. Watanabe, T. Taniguchi, D. H. Cobden, X. Xu, C. R. Dean, and M. Yankowitz, Electrically tunable correlated and topological states in twisted monolayer-bilayer graphene, arXiv:2004.11340.

[13] Y. Shi, S. Xu, M. M. Al Ezzi, N. Balakrishnan, A. GarciaRuiz, B. Tsim, C. Mullan, J. Barrier, N. Xin, B. A. Piot, T. Taniguchi, K. Watanabe, A. Carvalho, A. Mishchenko, A. K. Geim, V. I. Falko, S. Adam, A. H. Castro Neto, and K. S. Novoselov, Tunable van Hove singularities and correlated states in twisted trilayer graphene, arXiv:2004.12414.

[14] K.-T. Tsai, X. Zhang, Z. Zhu, Y. Luo, S. Carr, M. Luskin, E. Kaxiras, and K. Wang, Correlated superconducting and insulating states in twisted trilayer graphene Moiré of Moiré superlattices, arXiv:1912.03375.

[15] G. W. Burg, J. Zhu, T. Taniguchi, K. Watanabe, A. H. MacDonald, and E. Tutuc, Correlated Insulating States in Twisted Double Bilayer Graphene, Phys. Rev. Lett. 123, 197702 (2019).

[16] Y. Cao, D. Rodan-Legrain, O. Rubies-Bigorda, J. M. Park, K. Watanabe, T. Taniguchi, and P. Jarillo-Herrero, Tunable correlated states and spin-polarized phases in twisted bilayer-bilayer graphene, Nature (London) 583, 215 (2020).

[17] X. Liu, Z. Hao, E. Khalaf, J. Y. Lee, Y. Ronen, H. Yoo, D. H. Najafabadi, K. Watanabe, T. Taniguchi, A. Vishwanath, and P. Kim, Tunable spin-polarized correlated states in twisted double bilayer graphene, Nature (London) 583, 221 (2020).

[18] P. Rickhaus, F. de Vries, J. Zhu, E. Portoles, G. Zheng, M. Masseroni, A. Kurzmann, T. Taniguchi, K. Wantanabe, A. H. MacDonald, T. Ihn, and K. Ensslin, Density-wave states in twisted double-bilayer graphene, arXiv:2005.05373.

[19] P. C. Adak, S. Sinha, U. Ghorai, L. D. V. Sangani, K. Watanabe, T. Taniguchi, R. Sensarma, and M. M. Deshmukh, Tunable bandwidths and gaps in twisted double bilayer graphene on the verge of correlations, Phys. Rev. B 101, 125428 (2020).

[20] C. Shen, Y. Chu, Q. Wu, N. Li, S. Wang, Y. Zhao, J. Tang, J. Liu, J. Tian, K. Watanabe, T. Taniguchi, R. Yang, Z. Y. Meng, D. Shi, O. V. Yazyev, and G. Z. Shen, Correlated states in twisted double bilayer graphene, Nat. Phys. 16, 520 (2020).
[21] M. He, Y. Li, J. Cai, Y. Liu, K. Watanabe, T. Taniguchi, X. $\mathrm{Xu}$, and $\mathrm{M}$. Yankowitz, Tunable correlation-driven symmetry breaking in twisted double bilayer graphene, arXiv:2002.08904.

[22] X. Li, F. Wu, and A. H. MacDonald, Electronic structure of single-twist trilayer graphene, arXiv:1907.12338.

[23] Z. Ma, S. Li, Y.-W. Zheng, M.-M. Xiao, H. Jiang, J.-H. Gao, and X. C. Xie, Topological flat bands in twisted trilayer graphene, arXiv:1905.00622.

[24] Y.-H. Zhang, D. Mao, Y. Cao, P. Jarillo-Herrero, and T. Senthil, Nearly flat Chern bands in Moiré superlattices, Phys. Rev. B 99, 075127 (2019).

[25] C. Xu and L. Balents, Topological Superconductivity in Twisted Multilayer Graphene, Phys. Rev. Lett. 121, 087001 (2018).

[26] S. Carr, C. Li, Z. Zhu, E. Kaxiras, S. Sachdev, and A. Kruchkov, Ultraheavy and ultrarelativistic dirac quasiparticles in sandwiched graphenes, Nano Lett. 20, 3030 (2020).

[27] E. Khalaf, A. J. Kruchkov, G. Tarnopolsky, and A. Vishwanath, Magic angle hierarchy in twisted graphene multilayers, Phys. Rev. B 100, 085109 (2019).

[28] C. Mora, N. Regnault, and B. A. Bernevig, Flatbands and Perfect Metal in Trilayer Moiré Graphene, Phys. Rev. Lett. 123, 026402 (2019).

[29] A. C. Ferrari and D. M. Basko, Raman spectroscopy as a versatile tool for studying the properties of graphene, Nat. Nanotechnol. 8, 235 (2013).

[30] Z. Ni, L. Liu, Y. Wang, Z. Zheng, L.-J. Li, T. Yu, and Z. Shen, $G$-band Raman double resonance in twisted bilayer graphene: Evidence of band splitting and folding, Phys. Rev. B 80, 125404 (2009).

[31] R. W. Havener, H. Zhuang, L. Brown, R. G. Hennig, and J. Park, Angle-resolved Raman Imaging of interlayer rotations and interactions in twisted bilayer graphene, Nano Lett. 12, 3162 (2012).

[32] K. Kim, S. Coh, L. Z. Tan, W. Regan, J. M. Yuk, E. Chatterjee, M. F. Crommie, M. L. Cohen, S. G. Louie, and A. Zettl, Raman Spectroscopy Study of Rotated Double-Layer Graphene: Misorientation-Angle Dependence of Electronic Structure, Phys. Rev. Lett. 108, 246103 (2012).

[33] Y. Wang, Z. Su, W. Wu, S. Nie, N. Xie, H. Gong, Y. Guo, J. H. Lee, S. Xing, X. Lu, H. Wang, X. Lu, K. McCarty, S. Pei, F. Robles-Hernandez, V. G. Hadjiev, and J. Bao, Resonance Raman spectroscopy of G-line and folded phonons in twisted bilayer graphene with large rotation angles, Appl. Phys. Lett. 103, 123101 (2013).

[34] V. Carozo, C. M. Almeida, E. H. M. Ferreira, L. G. Cancado, C. A. Achete, and A. Jorio, Raman signature of graphene superlattices, Nano Lett. 11, 4527 (2011).

[35] J. Campos-Delgado, L. G. Cancado, C. A. Achete, A. Jorio, and J.-P. Raskin, Raman scattering study of the phonon dispersion in twisted bilayer graphene, Nano Res. 6, 269 (2013).

[36] R. He, T.-F. Chung, C. Delaney, C. Keiser, L. A. Jauregui, P. M. Shand, C. C. Chancey, Y. Wang, J. Bao, and Y. P. Chen, Observation of Low Energy Raman Modes in Twisted Bilayer Graphene, Nano Lett. 13, 3594 (2013).

[37] J.-B. Wu, X. Zhang, M. Ijas, W.-P. Han, X.-F. Qiao, X.-L. Li, D.-S. Jiang, A. C. Ferrari, and P.-H. Tan, Resonant 
Raman spectroscopy of twisted multilayer graphene, Nat. Commun. 5, 5309 (2014).

[38] O. Kashuba and V. I. Fal'ko, Signature of electronic excitations in the Raman spectrum of graphene, Phys. Rev. B 80, 241404 (2009).

[39] M. Mucha-Kruczynski, O. Kashuba, and V. I. Fal'ko, Spectral features due to inter-Landau-level transitions in the Raman spectrum of bilayer graphene, Phys. Rev. B 82, 045405 (2010).

[40] E. Riccardi, M.-A. Measson, M. Cazayous, A. Sacuto, and Y. Gallais, Gate-Dependent Electronic Raman Scattering in Graphene, Phys. Rev. Lett. 116, 066805 (2016).

[41] E. Riccardi, O. Kashuba, M. Cazayous, M.-A. Méasson, A. Sacuto, and Y. Gallais, Probing chiral electronic excitations in bilayer graphene by Raman scattering, Phys. Rev. Mater. 3, 014002 (2019).

[42] C. Faugeras, M. Amado, P. Kossacki, M. Orlita, M. Kuhne, A. A. L. Nicolet, Yu. I. Latyshev, and M. Potemski, Magneto-Raman Scattering of Graphene on Graphite: Electronic and Phonon Excitations, Phys. Rev. Lett. 107, 036807 (2011).

[43] P. Kossacki, C. Faugeras, M. Kuhne, M. Orlita, A. A. L. Nicolet, J. M. Schneider, D. M. Basko, Yu. I. Latyshev, and M. Potemski, Electronic excitations and electron-phonon coupling in bulk graphite through Raman scattering in high magnetic fields, Phys. Rev. B 84, 235138 (2011).

[44] Y. Henni, H. P. O. Collado, K. Nogajewski, M. R. Molas, G. Usaj, C. A. Balseiro, M. Orlita, M. Potemski, and C. Faugeras, Rhombohedral multilayer graphene: A magnetoRaman scattering study, Nano Lett. 16, 3710 (2016).

[45] A. García-Ruiz, S. Slizovskiy, M. Mucha-Kruczynski, and V. I. Fal'ko, Spectroscopic signatures of electronic excitations in raman scattering in thin films of rhombohedral graphite, Nano Lett. 19, 6152 (2019).

[46] A. García-Ruiz, M. Mucha-Kruczynski, and V. I. Fal'ko, Superconductivity-induced features in the electronic Raman spectrum of monolayer graphene, Phys. Rev. B 97, 155405 (2018).

[47] Yu. S. Ponosov, A. V. Ushakov, and S. V. Streltsov, Electronic Raman scattering in graphite and single-layer and few-layer graphene, Phys. Rev. B 91, 195435 (2015).

[48] M. Kuhne, C. Faugeras, P. Kossacki, A. A. L. Nicolet, M. Orlita, Yu. I. Latyshev, and M. Potemski, Polarizationresolved magneto-Raman scattering of graphenelike domains on natural graphite, Phys. Rev. B 85, 195406 (2012).

[49] C. Faugeras, P. Kossacki, A. A. L. Nicolet, M. Orlita, M. Potemski, A. Mahmood, and D. M. Basko, Probing the band structure of quadri-layer graphene with magneto-phonon resonance, New J. Phys. 14, 095007 (2012).

[50] S. Berciaud, M. Potemski, and C. Faugeras, Probing electronic excitations in mono- to pentalayer graphene by micro magneto-Raman spectroscopy, Nano Lett. 14, 4548 (2014).

[51] G. Li, A. Luican, J. M. B. Lopes dos Santos, A. H. Castro Neto, A. Reina, J. Kong, and E. Y. Andrei, Observation of Van Hove singularities in twisted graphene layers, Nat. Phys. 6, 109 (2010).
[52] J. M. Lopes Dos Santos, N. M. Peres, and A. H. Castro Neto, Graphene Bilayer with a Twist: Electronic Structure, Phys. Rev. Lett. 99, 256802 (2007).

[53] T. Stauber, P. San-Jose, and L. Brey, Optical conductivity, Drude weight and plasmons in twisted graphene bilayers, New J. Phys. 15, 113050 (2013).

[54] P. Moon and M. Koshino, Optical absorption in twisted bilayer graphene, Phys. Rev. B 87, 205404 (2013).

[55] R. Bistritzer and A. H. MacDonald, Moiré bands in twisted double-layer graphene, Proc. Natl. Acad. Sci. U.S.A. 108, 12233 (2011).

[56] E. McCann and M. Koshino, The electronic properties of bilayer graphene, Rep. Prog. Phys. 76, 056503 (2013).

[57] D. M. Basko, Theory of resonant multiphonon Raman scattering in graphene, Phys. Rev. B 78, 125418 (2008).

[58] H. Yoo, R. Engelke, S. Carr, S. Fang, K. Zhang, P. Cazeaux, S. H. Sung, R. Hovden, A. W. Tsen, T. Taniguchi, K. Watanabe, G.-C. Yi, M. Kim, M. Luskin, E. B. Tadmor, E. Kaxiras, and P. Kim, Atomic and electronic reconstruction at the van der Waals interface in twisted bilayer graphene, Nat. Mater. 18, 448 (2019).

[59] M. Koshino and E. McCann, Gate-induced interlayer asymmetry in ABA-stacked trilayer graphene, Phys. Rev. B 79, 125443 (2009).

[60] M. Koshino and E. McCann, Trigonal warping and Berrys phase $N \pi$ in ABC-stacked multilayer graphene, Phys. Rev. B 80, 165409 (2009).

[61] F. Guinea, A. H. Castro Neto, and N. M. R. Peres, Electronic states and Landau levels in graphene stacks, Phys. Rev. B 73, 245426 (2006).

[62] S. Slizovskiy, E. McCann, M. Koshino, and V. I. Fal'ko, Films of rhombohedral graphite as two-dimensional topological semimetals, Commun. Phys. 2, 164 (2019).

[63] H. Henck, J. Avila, Z. B. Aziza, D. Pierucci, J. Baima, B. Pamuk, J. Chaste, D. Utt, M. Bartos, K. Nogajewski, B. A. Piot, M. Orlita, M. Potemski, M. Calandra, M. C. Asensio, F. Mauri, C. Faugeras, and A. Ouerghi, Flat electronic bands in long sequences of rhombohedral-stacked graphene, Phys. Rev. B 97, 245421 (2018).

[64] See Supplemental Material at http://link.aps.org/ supplemental/10.1103/PhysRevLett.125.197401 for discussion of Hamiltonians for twisted double bilayers, examples of miniband spectra for all the four-layer twistronic graphene, and a model to approximate positions of the ERS peaks. Supplemental Material contains Refs. [55,59,60,65-67].

[65] D. A. Cosma, J. R. Wallbank, V. Cheianov, and V. I. Fal'ko, Moiré pattern as a magnifying glass for strain and dislocations in van der Waals heterostructures, Faraday Discuss. 173, 137 (2014).

[66] E. McCann and V. I. Fal'ko, Landau-Level Degeneracy and Quantum Hall Effect in a Graphite Bilayer, Phys. Rev. Lett. 96, 086805 (2006).

[67] A. H. Castro Neto, F. Guinea, N. M. R. Peres, K. S. Novoselov, and A. K. Geim, The electronic properties of graphene, Rev. Mod. Phys. 81, 109 (2009). 